\title{
Tuberculum sellae meningiomas
}

\author{
John H. Chi, M.D. M.P.H., And Michael W. MCDermotT, M.D. F.R.C.S.(C) \\ Department of Neurological Surgery, University of California, San Francisco, California
}

\begin{abstract}
Tuberculum sellae meningiomas are a classic tumor of the anterior fossa that present in patients with gradual visual deterioration secondary to optic apparatus compression. If untreated, complete blindness can occur. Treatment involves tumor removal and decompression of the optic chiasm via several operative approaches. Gross-total resection (Simpson Grade I or II) is the goal of treatment and can usually be accomplished safely. Special excision-related considerations include appreciation of arachnoid planes separating the tumor from neural tissue, adequate drilling of osseous elements for optimal exposure, and intraoperative preservation of the vascular supply to the optic apparatus. The authors reviewed their experience at the University of California, San Francisco, in cases of tuberculum sellae meningiomas treated between 1992 and 2002. In most patients, improvement of vision can be achieved with minimal postoperative complications and morbidity.
\end{abstract}

\section{KEY WORDS • tuberculum sellae • meningioma • anterior skull base • operative technique}

Meningiomas of the tuberculum sellae arise from the limbus sphenoidale, chiasmatic sulcus, and tuberculum. ${ }^{14,22}$ They comprise approximately 3 to $10 \%$ of all intracranial meningiomas. ${ }^{19,22}$ Correct diagnosis and management require an appreciation of the unique clinical, neuroimaging, and surgery-related features that distinguish these meningiomas from others of the anterior skull base. Tuberculum sellae meningiomas characteristically lie in a suprasellar subchiasmal midline position, displacing the optic chiasm posteriorly and slightly superiorly, and the optic nerves laterally. ${ }^{14}$ In cases involving large tumors extending toward the planum sphenoidale, sellae, and cavernous sinus, it can be difficult to differentiate tuberculum sellae meningiomas from others originating from the planum sphenoidale, olfactory groove, clinoid process, sellae, and medial sphenoid wing. Slowly progressing visual deterioration is the most common initial complaint, and prompt treatment is directed at preserving and improving vision. Management ideally consists of gross-total resection without injury to neighboring vital structures. To limit morbidity and mortality rates, knowledge of the anatomical relations of tuberculum sellae meningiomas is necessary.

\footnotetext{
Abbreviations used in this paper: $\mathrm{ACA}=$ anterior cerebral artery; $\mathrm{CT}=$ computerized tomography; $\mathrm{EBL}=$ estimated blood loss; $\mathrm{MR}=$ magnetic resonance.
}

\section{REVIEW OF SURGICAL TREATMENT}

\section{Clinical Presentation and Demographic Features}

Visual deterioration is the most common problem in patients harboring tuberculum sellae meningiomas. Patients typically present with a history of uni- or bilateral visual decline progressing over months to years. Visual deterioration seems to be present early and often begins unilaterally. Because growth is slow and insidious in most cases, evaluation and diagnosis are typically delayed, and significant vision loss of one or both eyes occurs. As such, pre- and postoperative ophthalmological evaluation for both visual acuity and visual fields is mandatory. Humphrey visual fields are standard in the pre- and postoperative evaluation of these patients. If evaluation is delayed tumors can grow and cause optic chiasm compression, which is less common today because of earlier diagnosis. On examination, greater than $95 \%$ of patients suffer visual acuity and/or field deficits and approximately 75 to $90 \%$ have optic atrophy. ${ }^{1-3,6,19}$ The pattern of vision loss can vary, and although a bitemporal hemianopsia has been described as the most frequent field cut observed, ${ }^{1}$ this has not been corroborated in recent series. Quadrantanopsia and unilateral temporal field defects are commonly reported in patients who have undergone formal ophthalmological visual field testing. ${ }^{3,8}$

Headache is the second most common associated symp- 
tom occurring in approximately 35 to $45 \%$ of patients, and it may occasionally be the only presenting symptom. ${ }^{4,10}$ Facial numbness, altered mental status, seizures, endocrinopathies, and anosmia have all been reported in association with tuberculum sellae meningiomas but are exceedingly rare.

Evidence of endocrinopathies at presentation is present in only a small number of patients. Reduced libido, alteration in menses, and elevated prolactinemia are found in 5 to $10 \%$ of patients, but clear evidence of endocrinopathies should suggest a diagnosis of pituitary adenoma rather than meningioma. ${ }^{2}$ Diabetes insipidus, however, is encountered in cases involving meningiomas originating from the diaphragma sellae, and serum sodium and pituitary function tests should be performed in all patients. ${ }^{2,9}$ Preoperative differentiation between tuberculum and diaphragma sellae meningiomas may be difficult to make based on clinical features or neuroimaging findings, and diagnosis is made intraoperatively when dural attachment to the diaphragma is visualized. Unilateral weakness has also been reported, usually in the presence of significant frontal lobe edema, and bilateral frontal lobe involvement can manifest with disinhibition and behavioral changes.

Tuberculum sellae meningiomas are more common in women than men, and diagnosis is typically made in the fifth to sixth decade of life.

\section{Diagnostic Imaging}

Although tuberculum sellae meningiomas follow the typical CT and MR imaging characteristics of meningiomas in general, in this region they can easily be confused for pituitary adenoma. Meningiomas appear isodense to brain on CT scans and generally isointense to brain on $\mathrm{T}_{1}-$ weighted and hypointense on $\mathrm{T}_{2}$-weighted on MR images, whereas pituitary adenomas tend to demonstrate a higher $\mathrm{T}_{2}$-weighted signal. After administration of intravenous contrast, meningiomas produce a homogeneous contrast enhancement (Fig. 1), whereas adenomas demonstrate patchy enhancement. Although hyperostosis and intrasellar calcifications on CT may suggest meningioma, pituitary adenoma or craniopharyngioma can not be ruled out. The sellae turcica is usually not expanded or only slightly enlarged in tuberculum sellae meningiomas, in contrast to pituitary adenomas, which usually expand the sellae. Additionally, a suprasellar base, optic canal extension, and a

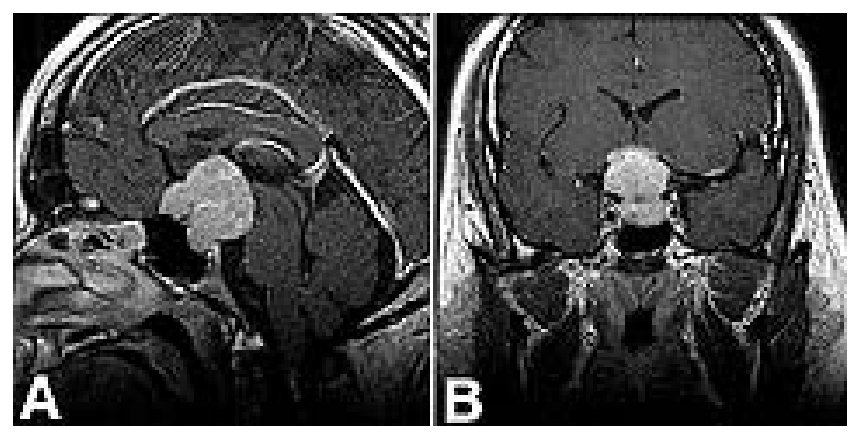

Fig. 1. Gadolinium-enhanced sagittal (A) and coronal (B) $\mathrm{T}_{1}{ }^{-}$ weighted MR images demonstrating a tuberculum sellae meningioma with suprasellar and sellar involvement. tapered dural tail are both suggestive of meningioma rather than pituitary adenoma. ${ }^{21}$ Other lesions in the differential diagnosis include optic glioma, sarcoidosis, aneurysm, craniopharyngioma, and metastasis.

Angiographic embolization of tuberculum sellae meningiomas is not routinely performed. The vascular supply tends to derive from small perforating branches from the posterior ethmoidal, ophthalmic, superior hypophysial, and $\mathrm{A}_{1} / \mathrm{A}_{2}$ artery segments. Preoperative angiography can help demonstrate distorted vascular anatomy secondary to tumor mass effect, which usually reveals posterior displacement of the $A_{1}$ and $A_{2}$ segments of the ACA in $80 \%$ of patients and encasement of the $A_{1}$ segment in $24 \%{ }^{6,22}$

\section{Surgical Options}

Although controversy exists as to the best surgical approach, in truth several approaches may be suitable for a variety of tumor sizes and attachments in this area. The pterional, unilateral subfrontal, and orbitofrontal approaches may be used alone or in combination for smallto medium-sized tumors, whereas a bifrontal approach may be performed for larger tumors. We have come to appreciate the flexibility and limited morbidity rate associated with the bifrontal extended frontal craniotomy to reach tumors of this region. The bifrontal subfrontal exposure provides excellent views of the entire tuberculum sellae and proximal medial portion of the optic canal; it also allows the option of swinging laterally to open the proximal sylvian fissure to identify the optic nerves displaced by tumor. The supraorbital osteotomy greatly reduces the extent of frontal retraction, limiting postoperative neuropsychological sequelae.

\section{Surgical Approach: Surgeon's Perspective}

Prior to surgery, Humphrey visual fields should be documented to allow for postoperative comparisons. Confrontation eye fields provide an inadequate assessment. Axial and coronal fat-suppressed MR images should be reviewed to determine the extent of optic canal involvement and whether there is hyperostosis and osseous infiltration of the tuberculum sellae. Midsagittal images will always demonstrate a component of the tumor extending down over the tuberculum sellae to the superior aspect of the pituitary gland. Typically, this component cannot be adequately resected unless the tuberculum sellae is drilled off, and blind scraping with pituitary curettes is potentially injurious to the pituitary stalk and/or medial portions of the optic nerves.

At surgery a lumbar subarachnoid drain is inserted for cerebrospinal fluid drainage. The patient is positioned supine on the operating room table, with the neck flexed on the chest and the head extended on the neck. Draping for a bicoronal incision is performed with care not to place draping towels above the eyebrows because this will create too much pressure for the skin when the scalp flap is turned forward. A small quadrant of the abdomen is prepared for harvesting of a fat graft to cover the sphenoid sinus mucosa when the tuberculum sellae is drilled out.

A standard bicoronal scalp incision is turned down and care is taken not to incise the pericranium. The pericranium is elevated off the superior temporal lines bilaterally to the supraorbital margins. Supraorbital nerves are dissect- 
ed from their notches of foramina, and the periorbita is dissected from the roof and lateral walls of the orbit. The pericranium is reflected past the nasofrontal suture. The temporalis muscles are released from the superior temporal line, leaving a cuff of muscle to reattach to at closure. The bifrontal bone flap is then elevated and the dura mater dissected from the roof of the orbits bilaterally. In the midline the dura mater should not be reflected posterior to the crista galli to avoid damaging the olfactory nerves. With the extradural dissections completed, an oscillating saw is used to make cuts at the level of the frontozygomatic suture laterally, continuing from the cranial side through the roofs of the orbits in front of the crista galli. Close to the midline, the roof of the frontal sinus is entered so that the tip of the blade cannot be seen from the orbital side. Usually the limit for the orbital cut is a short distance down the medial side of the orbit, and these two bone cuts are connected using the oscillating saw and cutting down on the nasofrontal suture. The supraorbital osteotomy bone piece can then be removed and the mucosa stripped from frontal sinus recesses on both the craniotomy flap and supraorbital osteotomy to prevent development of mucoceles in the late follow-up period. The exposed frontal sinus is then plugged using gelfoam and covered with $0.5 \times 3-$ [cm, mm, inches?] cottonoids.

The dura is then opened a finger's breadth above the orbits and the dura sutured forward. The superior sagittal sinus is suture ligated, and the falx cut back through its free edge inferiorly. Rubber dams are used to cover the inferior frontal lobes, and the olfactory tracts are then dissected using the operating microscope posteriorly to the medial and lateral striae and covered with small thin rubber dams. Unless the nerves are covered, they will become dry and nonfunctional by the end of the procedure. The olfactory tract can be spared bilaterally in cases involving small tumors and often unilaterally even in cases of larger ones. We use a single retractor blade, directing it as needed for the appropriate exposure. In this way, prolonged retraction over any one area is avoided.

Once the tumor is identified, the first objective is to determine the position of the optic nerves. For very large tumors this cannot be established until the tumor is debulked. This is begun in the midline, taking down the tuberculum sellae attachments and working straight back in and just off the midline. Once the optic nerves are identified, the tumor is debulked to allow dissection of the arachnoid plane separating the nerve and tumor. Great care must be taken not to damage the small superior hypophysial branches arising from the medial wall of the carotid artery supplying the optic chiasm and stalk. The $\mathrm{A}_{1}$ segments of the ACA and the anterior communicating artery will be seen and must be dissected from the surface of large tumors. Branches of the medial orbital frontal and frontopolar arteries will supply larger tumors.

Once the majority of the tumor has been removed, we attend to the region of the optic canal and tuberculum sellae. Tumors will frequently extend down the proximal medial aspect of the optic canal attach at its junction with the chiasmatic sulcus. The falciform ligament can be cut and the roof of the optic canal drilled out to ensure tumor removal. To remove tumor extending over the tuberculum sellae, this bone should be resected. The dura is incised from the optic canals bilaterally and reflected back over the tuberculum sellae. A diamond drill is then used to drill down the tuberculum sellae to the level of the anterior intercavernous sinus. Once the sphenoid sinus mucosa is exposed, care is taken to keep it intact by dissecting it away from bone and covering it with a small cottonoid. Once the tuberculum sellae is removed, the dural cuff is excised, exposing any remaining tumor and the diaphragma sellae. At the completion of tumor excision, fibrin glue is used to secure a small fat graft over the sinus opening.

Once the dura is closed, the pericranium is reflected over the exposed frontal sinus and the supraorbital osteotomy bone piece is then resecured using plates and screws. The remaining closure is completed in standard fashion.

\section{CLINICAL MATERIAL AND METHODS}

Between January 1992 and January 2003, 21 patients underwent resection of meningiomas originating from the tuberculum sellae at the University of California, San Francisco. The lesion was diagnosed by preoperative neuroimaging and operative report findings. Approval by the Committee on Human Research was obtained and inpatient charts, operative reports, neuroimaging studies, and clinical and ophthalmological data were reviewed retrospectively. One sample surgery was videotaped for the purposes of demonstrating the microsurgical technique. All patients underwent postoperative MR imaging to confirm the extent of resection.

\section{RESULTS}

\section{Demographics and Presenting Symptoms}

In our series, the mean age at diagnosis was 52.3 years (range 28-80 years). The female/male ratio was 2:1. There were no ethnic or racial propensities. The most common presenting symptom was vision loss in $90 \%$ of patients. Reduced visual acuity was found in $75 \%$, whereas formal visual field testing showed defects in $80 \%$ of patients. Headache was also common (35\% of cases); it occurred as the only presenting symptom in 10\%. Diabetes insipidus was present in one patient (5\%) and facial numbness, along with visual decline and headache, were also reported in one patient $(5 \%)$. Formal ophthalmological testing demonstrated visual deficits in all patients, either decreased acuity or field cuts, and bilateral involvement was more common than unilateral involvement alone. The mean duration of symptoms at the time of evaluation and diagnosis was 18 months (range 2 months-5 years) (Table 1).

\section{Surgical Approaches and Operative Variables}

The bifrontal extended craniotomy was the preferred approach in our series (11 patients [52.4\%]). The unilateral subfrontal approach was undertaken in eight patients $(38 \%)$, whereas a pterional and transsphenoidal route were chosen for one patient each. The mean operative time was 10.2 hours (range 4-16 hours for craniotomies). In the one case requiring a transsphenoidal approach, the operative time was only 3 hours. The mean EBL was 552 


\section{J. H. Chi and M. W. McDermott}

TABLE 1

Preoperative characteristics in 21 patients with tuberculum sellae meningiomas

\begin{tabular}{cc}
\hline \hline Characteristic & No. of Cases $(\%)$ \\
\hline demographics & \\
age (yrs) & \\
mean 52.3 & \\
range 28-80 & \\
sex female & $14(66.7)$ \\
male & $7(33.3)$ \\
symptom & $15(75)$ \\
blurry vision & $16(80)$ \\
field cut & $7(35)$ \\
headache & $1(5)$ \\
endocrine dysfunction & $1(5)$ \\
facial numbness & $2(10)$ \\
headache alone & \\
visual deficit involvement & $9(45)$ \\
unilat predominant & $11(55)$ \\
bilat predominant & \\
duration of symptoms & \\
mean 18 mos & \\
range 2 mos-5 yrs & \\
\hline
\end{tabular}

$\mathrm{ml}$ (range 300-2000 $\mathrm{ml}$ for craniotomy; $50 \mathrm{ml}$ for transsphenoidal). Blood transfusions were only required in two cases in which EBL exceeded $1 \mathrm{~L}$ (Table 2).

\section{Outcome Data}

The extent of Simpson Grade-based resection ${ }^{18}$ was judged according to the primary surgeon's operative report. A gross-total resection (Simpson Grade I-III) was achieved in 66.5\%, whereas subtotal resections (Simpson Grade IV-V) were identified in the remaining patients. The most common Simpson grade was II (33\%) followed by I and IV (28.5\% each). All patients in whom subtotal resection was performed were referred for external-beam radiotherapy.

The mean length of hospital stay was 6.15 days for craniotomy-treated patients who were discharged to home or rehabilitation. The shortest stay was 3 days in a patient in whom the transsphenoidal approach was used. Eighteen patients $(85 \%)$ were discharged to home, whereas two patients $(10 \%)$ were sent for acute rehabilitation before returning home. Only one patient was sent to comfort care following a prolonged intensive care unit course complicated by the development of sepsis and multiorgan failure. There were no deaths attributed to surgery directly.

In a mean follow-up period of 3 months, clear improvement in vision was demonstrated in $47 \%$ of patients and preserved vision with no further deterioration in $42 \%$. Two patients (11\%) experienced worsened vision following surgery. There were no cases of new endocrinological deficits (Table 3 ).

\section{DISCUSSION}

The tuberculum sellae is a slight osseous protuberance between the chiasmatic sulcus and the anterosuperior limit of the pituitary fossa. The diaphragma of the sella is a layer of dura stretching from the tuberculum over the sella
TABLE 2

Operative characteristics in 21 patients undergoing resection of meningiomas

\begin{tabular}{cc}
\hline \hline Characteristic & No. of Cases $(\%)$ \\
\hline surgical approach & $11(52.4)$ \\
bifrontal extended & $8(38)$ \\
unilat subfrontal & $1(5)$ \\
pterional & $1(5)$ \\
transphenoidal & \\
grade of resection* & $6(28.5)$ \\
I & $7(33)$ \\
II & $1(5)$ \\
III & $6(28.5)$ \\
IV & $1(5)$ \\
V & \\
op time (hrs) & \\
mean 10.2 & \\
range 4-16 & \\
EBL (ml) & \\
mean 552 & \\
range 50-2000 & \\
\hline Based on the Simpson grading scheme. &
\end{tabular}

turcica to the posterior clinoids, creating a roof over the pituitary fossa. The limbus sphenoidale is identified as a small borderline separating the planum sphenoidale and chiasmatic sulcus. The dural attachment of tuberculum sellae meningiomas may arise and extend onto all of these structures. The tuberculum sellae is bounded laterally by the clinoid processes, internal carotid and posterior communicating arteries with the arachnoid of the carotid cisterns, posteriorly by the pituitary stalk, infundibulum, and Liliequist membrane and superiorly by the optic chiasm, lamina terminalis, and ACA complex. ${ }^{5,17}$ This space is quite small, (mean length $8 \mathrm{~mm}$, mean width $11 \mathrm{~mm}$ ), ${ }^{8}$ and the path of least resistance for tumor growth tends to be over the planum sphenoidale anteriorly (perhaps due to a defect in the chiasmatic cistern arachnoid), around the optic nerves sometimes into the optic canals laterally, above the chiasm, displacing it superiorly, and down over the tuberculum and sella inferiorly. In essence, a layer of arachnoid is always present between tumor surrounding brain structures, and this provides a plane of dissection to aid the surgeon. Because of the limits of the tuberculum

TABLE 3

Outcomes in 21 patients after resection of tuberculum sellae meningioma

\begin{tabular}{cc}
\hline \hline Factor & No. of Cases $(\%)$ \\
\hline disposition & \\
home & $18(85)$ \\
acute rehab & $2(10)$ \\
comfort care & $1(5)$ \\
deaths & 0 \\
post op vision* & $9(47)$ \\
improved & $8(42)$ \\
same & $2(11)$ \\
worsened & \\
length of stay 6.15 days & \\
\hline
\end{tabular}

* Determined at 3-month follow-up evaluation. 
sellae, meningiomas in this region never extend into the posterior fossa.

The overall morbidity and mortality rate associated with resection of tuberculum sellae meningiomas is quite low. Preservation of vision is the most important goal of treatment, and several factors have been shown to affect outcome. Improvement in vision has been reported in 40 to $80 \%$ of patients undergoing surgery. ${ }^{1,2,23}$ No change in visual status has been reported in 18 to $40 \%$, whereas worsened vision has been reported in 17 to $20 \%$. $^{2,4,12}$ The follow-up periods and validity of vision assessment vary among studies; however, as a whole, improvement or preservation of vision is more common than the development of new visual deficits in patients undergoing surgery for tuberculum sellae meningiomas. Most authors agree that the key to success in preserving vision is adhering to arachnoid planes around the optic nerves, chiasm, and brain parenchyma, as well as recognizing that the vascular supply to the optic apparatus is derived via perforating branches off the posterior ethmoidal, superior hypophysial, and ACAs. Patients in whom the tumor is subtotally resected should be referred for external-beam radiotherapy; the efficacy of newer modalities such as radiosurgery and intensity modulated radiotherapy are being investigated. ${ }^{7}$

The authors of several studies have demonstrated that poor preoperative visual acuity predicts less visual improvement after decompressive surgery. ${ }^{1-3,8,13}$ Such deficits do not preclude aggressive treatment in patients with poor visual function because some improvement often occurs and progression of visual deficit is halted. Age also serves as a predictor of outcome, with older age predisposing to less posttreatment improvement. Several age limits, 54 and 64 years of age, ${ }^{16,23}$ have been suggested, but these parameters are arbitrary. The duration of visual symptoms prior to diagnosis also predicts outcome. The authors of several case series have shown that patients with symptoms present lasting fewer than 6 months tend to recover more visual function than those with symptom duration longer than 1 year. ${ }^{1,6}$ Fahlbusch and Schott $^{2}$ reported that $100 \%$ of their patients in whom symptoms were present fewer than 6 months prior to diagnosis experienced improvement in vision, whereas those with symptoms lasting 6 to 12 months and those with symptoms lasting longer than 12 months experienced improved vision in 83 and $60 \%$, respectively, during follow up. Interestingly, tumor size does not affect outcome. Although historically it has been reported that suprasellar tumor size greater than $3 \mathrm{~cm}$ negatively affects outcome, ${ }^{15,19}$ more series do not support this. ${ }^{3,6,8,16,23}$ Additionally, Simpson grade and extent of involvement of surrounding structures (such as arteries and chiasm) does not seem to affect outcome in any predictable fashion. ${ }^{11,23}$ Notably, diaphragma sellae meningiomas tend to be associated with higher rates of endocrinological effects, namely diabetes insipidus. ${ }^{9}$ Some investigators have proposed a separate categorization of these tumors based on clinical presentation and anatomical features, but such distinction cannot be made preoperatively based on current neuroimaging techniques.

In our series of 21 patients with tuberculum sellae meningiomas, we found that vision-related outcome rates were similar to those reported in prior studies. Cases of improved and preserved vision far outnumber those of worsened vision. In 47,42 , and $11 \%$ of our patients, vision status improved, stabilized, and worsened, respectively. True assessments of visual outcome can only be made by conducting quantitative testing of visual fields and light intensity thresholds, such as by Humphrey visual field testing. We perform this testing preoperatively and then at 3 and 6 months postoperatively. Those patients treated for residual or recurrent disease with radiotherapy should undergo repeated visual fields testing annually.

\section{CONCLUSIONS}

Tuberculum sellae meningiomas should be resected early in their course in patients presenting with symptoms of optic chiasm compression. Adequate preoperative visual field assessment is necessary to allow for objective evaluation of postoperative visual results. Although there are several surgical approaches to these tumors, we believe that the bifrontal extended frontal approach yields minimal morbidity and allows the surgeon the greatest intraoperative flexibility for dissection and tumor removal. Drilling down the tuberculum and excising the involved dura is the only way to be certain of total tumor removal. With modern microsurgical techniques and a thorough knowledge of the microvascular anatomy of the blood supply to the optic nerves and chiasm, excellent visual outcomes can be achieved.

\section{References}

1. Andrews BT, Wilson CB: Suprasellar meningiomas: the effect of tumor location on postoperative visual outcome. J Neurosurg 69:523-528, 1988

2. Fahlbusch R, Schott W: Pterional surgery of meningiomas of the tuberculum sellae and planum sphenoidale: surgical results with special consideration of ophthalmological and endocrinological outcomes. J Neurosurg 96:235-243, 2002

3. Goel A, Muzumdar D, Desai KI: Tuberculum sellae meningioma: a report on management on the basis of a surgical experience with 70 patients. Neurosurgery 51:1358-1364, 2002

4. Gokalp HZ, Arasil E, Kanpolat Y, et al: Meningiomas of the tuberculum sella. Neurosurg Rev 16:111-114, 1993

5. Gray H: Anatomy of the Human Body, ed 30. Philadelphia: Lea \& Febiger, 1985

6. Grisoli F, Diaz-Vasquez P, Riss M, et al: Microsurgical management of tuberculum sellae meningiomas. Results in 28 consecutive cases. Surg Neurol 26:37-44, 1986

7. Iwai Y, Yamanaka K, Nakajima H: The treatment of skull base meningiomas-combining surgery and radiosurgery. J Clin Neurosci 8:528-533, 2001

8. Jallo GI, Benjamin V: Tuberculum sellae meningiomas: microsurgical anatomy and surgical technique. Neurosurgery 51: 1432-1440, 2002

9. Kinjo T, Al-Mefty O, Ciric I: Diaphragma sellae meningiomas. Neurosurgery 36:1082-1092, 1995

10. Leu CH, Hu TL, Shen CC, et al: Tuberculum sellae meningiomas: clinical manifestation, radiologic diagnosis, surgery and visual outcome. Zhonghua Yi Xue Za Zhi 61:1-7, 1998

11. Ohta K, Yasuo K, Morikawa M, et al: Treatment of tuberculum sellae meningiomas: a long-term follow-up study. J Clin Neurosci (Suppl 1) 8:26-31, 2001

12. Puchner MJ, Fischer-Lampsatis RC, Herrmann HD, et al: Suprasellar meningiomas - neurological and visual outcome at long-term follow-up in a homogeneous series of patients treated microsurgically. Acta Neurochir 140:1231-1238, 1998

13. Raco A, Bristot R, Domenicucci M, et al: Meningiomas of the 
tuberculum sellae. Our experience in 69 cases surgically treated between 1973 and 1993. J Neurosurg Sci 43:253-262, 1999

14. Rengachary SS, Wilkins RH: Neurosurgical Operative Atlas. Park Ridge, IL: AANS Publications: Baltimore: Williams \& Wilkins, Vol 1, 1991

15. Rosenstein J, Symon L: Surgical management of suprasellar meningioma. Part 2: Prognosis for visual function following craniotomy. J Neurosurg 61:642-648, 1984

16. Rubin G, Ben David U, Gornish M, et al: Meningiomas of the anterior cranial fossa floor. Review of 67 cases. Acta Neurochir 129:26-30, 1994

17. Seeger W: Atlas of Topographical Anatomy of the Brain and Surrounding Structures for Neurosurgeons, Neuroradiologists, and Neuropathologists. New York: Springer-Verlag, 1978

18. Simpson D: The recurrence of intracranial meningiomas after surgical treatment. J Neurol Neurosurg Psychiatry 20:22-39, 1957

19. Sklar EM, Schatz NJ, Glaser JS, et al: Optic tract edema in a meningioma of the tuberculum sellae. AJNR 21:1661-1663, 2000

20. Symon L, Rosenstein J: Surgical management of suprasellar meningioma. Part 1: The influence of tumor size, duration of symptoms, and microsurgery on surgical outcome in 101 consecutive cases. J Neurosurg 61:633-641, 1984

21. Taylor SL, Barakos JA, Harsh GR IV, et al: Magnetic resonance imaging of tuberculum sellae meningiomas: preventing preoperative misdiagnosis as pituitary macroadenoma. Neurosurgery 31:621-627, 1992

22. Youmans JR: Neurological Surgery: A Comprehensive Reference Guide to the Diagnosis and Management of Neurosurgical Problems, ed 4. Philadelphia: WB Saunders, Vol 3, 1996

23. Zevgaridis D, Medele RJ, Muller A, et al: Meningiomas of the sellar region presenting with visual impairment: impact of various prognostic factors on surgical outcome in 62 patients. Acta Neurochir 143:471-476, 2001

Manuscript received May 23, 2003.

Accepted in final form June 4, 2003.

Address reprint requests to: John Chi, M.D., M.P.H., 505 Parnassus Avenue, M-779 Box 0112, University of California, San Francisco, San Francisco, California 94143. email: chijo@ neurosurg.ucsf.edu. 